\title{
Degradation of Cellulose in a Vacuum with Ultraviolet Light
}

\author{
Joseph H. Flynn, William K. Wilson, and William L. Morrow
}

\begin{abstract}
Dried, purified, cotton cellulose sheets were irradiated in a vacuum at $40^{\circ} \mathrm{C}$ with light of 2537 A wavelength. Hydrogen, carbon monoxide, and carbon dioxide were evolved, the degree of polymerization (D. P.) decreased, and $\mathrm{CHO}$ and $\mathrm{COOH}$ groups were produced. The evolution of hydrogen has not been reported previously. The rate of hydrogen evolution followed a parabolic rate law indicating inhibition by a product. The rate of evolution of carbon monoxide plus carbon dioxide increased slightly during irradiation. The initial quantum yield was $10^{-2}$ for hydrogen and $10^{-3}$ for carbon monoxide and carbon dioxide. A mechanism is proposed in which alcohol groups are photolyzed to carbonyl with the liberation of hydrogen.
\end{abstract}

\section{Introduction}

The study of the irradiation in a vacuum of polymeric materials that contain specific functional groups holds several advantages over the gas-phase photochemical studies of smaller organic compounds containing such groups. In the former, the gaseous dissociation products of the primary process may be allowed to diffuse from the system to a collection point. The reactions of labile dissociation products attached to the solid are minimized due to their immobility, and these products may be characterized to some extent after exposure to various gaseous atmospheres. Thus, complicating kinetic chains can be eliminated and the interaction of reactants, intermediates, and products can be kept at a minimum.

Cellulose is a substrate that is particularly adaptable to this type of study. Celluloses containing various amounts of carboxyl, aldehyde, and other groups are easily prepared, and quantitative procedures are available for determining concentrations of these groups before and after irradiation.

The photochemical degradation of cellulose has been extensively investigated as it is an important cause of deterioration of textiles and paper. Past investigators have found that the irradiation of cellulose with 2537 A light results in considerable degradation both in the presence and absence of oxygen. Increase in carboxyl and reducing groups upon exposure to air after irradiation has been noted, carbon monoxide and carbon dioxide have been observed as gaseous products, and decrease in glucosidic linkages has been evidenced by loss in strength, decrease in percent alpha-cellulose, and decrease in degree of polymerization (D. P.) [1]. ${ }^{1}$

The amount of degradation was independent of the presence of oxygen during irradiation and was inhibited by water vapor [2]. A post-irradiation decomposition took place at a very low rate in the presence of oxygen [2,3], suggesting the presence of labile dissociation products in the irradiated polymer.

This paper describes the irradiation of purified, dried, cotton cellulose in an evacuated system with 2537 A light. The composition and rate of evolution of gaseous products were measured and changes in functional group content and degree of polymerization of the irradiated cellulose were determined.

\footnotetext{
1 Figure in brackets indicate the literature references at the end of this paper.
}

\section{Experimental Procedure}

\subsection{Preparation of Cellulose}

Clean cotton sliver was purified by a modification of the method of Warner and Mease [4]. The purified cotton was cut into quarter-inch lengths, beaten with the minimum amount of distilled water in an agate mortar, and made into sheets on a British Handsheet Machine. These sheets were cut to form rectangles of $92 \mathrm{~cm}^{2}, 0.0018-\mathrm{cm}$ thick and weighing $0.86 \mathrm{~g}$. These were dried by occasional heating at $100^{\circ} \mathrm{C}$ in the irradiation apparatus for over a week until the system would maintain a pressure of less than $10^{-6} \mathrm{~mm}$ of $\mathrm{Hg}$.

\subsection{Irradiation of Cellulose}

The reaction vessel and irradiation apparatus are shown in figure 1 . The cellulose sheet was placed around a cold finger through which water from a constant-temperature bath was circulated. The cold finger was sealed into a large quartz tube which was

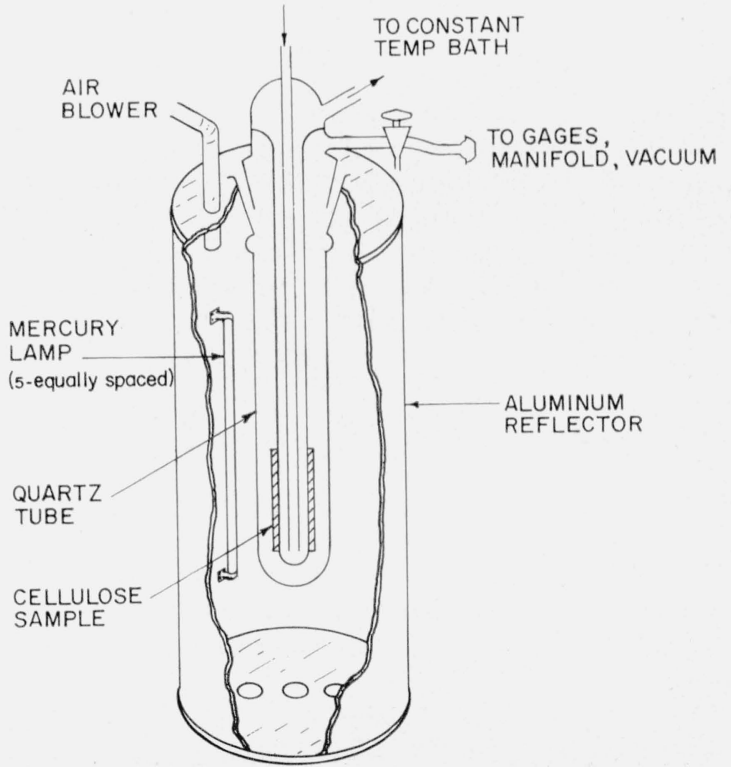

FIGURE 1. Irradiation apparatus. 
inserted axially into the irradiation apparatus and connected by a stopcock to the vacuum manifold.

The cellulose was irradiated by five Hanovia quartz low-pressure mercury lamps spaced evenly about the interior of an etched cylindrical aluminum reflector. The lamps were controlled by separate Variacs and transformers, and their operating temperature was maintained at $50^{\circ} \pm 1^{\circ} \mathrm{C}$ by an air blower, as their output is quite dependent on temperature [5]. Under these conditions, the energy in the ultraviolet region results almost entirely from the $2537 \mathrm{~A} \mathrm{Hg}$ line. The intensity of the irradiating light was calculated from periodic calibration of the lamps in place in the reflector by a selenium photovoltaic cell with a Willemite converter. This sensing device was calibrated against a standard 2537 A source by the Radiometry Section of the Bureau and used in its most sensitive range.

The pressure of evolved gas was measured by a thermocouple gage and a discharge gage of the RCA Vacuum Gage Unit, Type EMG-1, and by a McLeod mercury manometer.

\subsection{Analysis of Gaseous Products}

The composition of gaseous products was determined with a mass spectrometer. Also, the percent hydrogen of the gases in the system was estimated by comparison of the thermocouple and McLeod gage readings.

\subsection{Analysis of the Irradiated Cellulose}

The irradiated cellulose was stored for several months in air and was analyzed to determine changes in the degree of polymerization, carboxyl content, and aldehyde content.

\section{a. Degree of Polymerization (D. P.)}

The weight-average D. P. was calculated from cuprammonium viscosity data using a relation developed by Battista [6]. The cuprammonium solvent was prepared by the method of Launer and Wilson [7]. The cellulose was dissolved in the cuprammonium solvent in glass vessels containing copper and filled with solvent to exclude air. The viscosities of the 0.50 percent by weight cellulose solutions were determined with Ostwald-Cannon-Fenske viscometers calibrated with NBS standard oils.

\section{b. Carboxyl Content}

The carboxyl content was determined by a modification of Davidson's methylene blue adsorption method [8]. Automatic 1-ml pipets coated with Dow Corning 200 fluid were used to deliver the centrifuged methylene blue solutions to volumetric flasks for dilution. A Beckman Model DU Quartz Spectrophotometer was used for the absorbance measurements.

\section{c. Aldehyde Content}

Aldehyde was determined by oxidation of the irradiated cellulose with a solution of sodium chlorite in $\mathrm{HOAc}-\mathrm{NaOAc}$ buffer $(p \mathrm{H}=3.5$, ionic strength $=$ 0.11 ) at $40^{\circ} \mathrm{C}$ followed by determination of the amount of chlorite consumed [9]. In calculating the aldehyde content, an equation developed from the kinetics of the oxidation of glucose with this reagent was used [10].

\subsection{Reflectance}

The refleotance of the cellulose sheets was measured by comparison with a fresh surface of magnesium carbonate. The latter reflects 72 percent of the 2537 A radiation [11]. The cellulose sheets initially reflected 55 percent and transmitted 1.8 percent of the 2537 A light.

\section{Results}

\subsection{Gaseous Products}

Hydrogen, carbon monoxide, and carbon dioxide were evolved during the irradiation. The mole percent of hydrogen decreased with increasing dosage and could be extrapolated to an initial value of 92 percent. Table 1 gives the results of the mass spectrometric analysis of gases evolved during an irradiation of $2.84 \times 10^{-4} N h \nu / \mathrm{cm}^{2}$ at $40^{\circ} \mathrm{C}$ where $N$ is Avogadro's number, $h$ is Planck's constant, and $\nu$ is the frequency of the irradiating light.

TABLE 1. Gaseous products from irradiation of cellulose in a vacuum

\begin{tabular}{|c|c|c|c|}
\hline & \multicolumn{3}{|c|}{ Component } \\
\cline { 2 - 4 } & $\mathrm{H}_{2}$ & $\mathrm{CO}$ & $\mathrm{CO}_{2}$ \\
\hline Mole percent__... & 87.5 & 8.2 & 4.3 \\
\hline
\end{tabular}

The rate of hydrogen evolution fell off with increasing dosage but behaved erratically at higher total gas pressure. In order to minimize the reaction between gaseous products and the irradiated cellulose, the system was alternately isolated and evacuated for 5-min periods during the irradiation for many of the runs. Thus, the total gas pressure was kept below $0.03 \mathrm{~mm}$. A plot of the square of the reciprocal of the rate of hydrogen evolution versus dosage (time) in figure 2 gave a linear relationship, indicating conformance to the parabolic rate law [12] given in eq (1):

$$
\frac{d x}{d t}=\frac{k}{1+a x}
$$

where $t=$ time, $x=$ moles of hydrogen formed, and both $k$, the initial rate, and $a$ are constants. A plot of $\log k$ for a particular cellulose sheet versus log intensity resulted in a straight line of unit slope for intensities of

$13.4 \times 10^{-9}, 9.62 \times 10^{-9}$, and $6.49 \times 10^{-9} \mathrm{Nhv} / \mathrm{cm}^{2}$ sec.

The intensities recorded in this paper and used in 


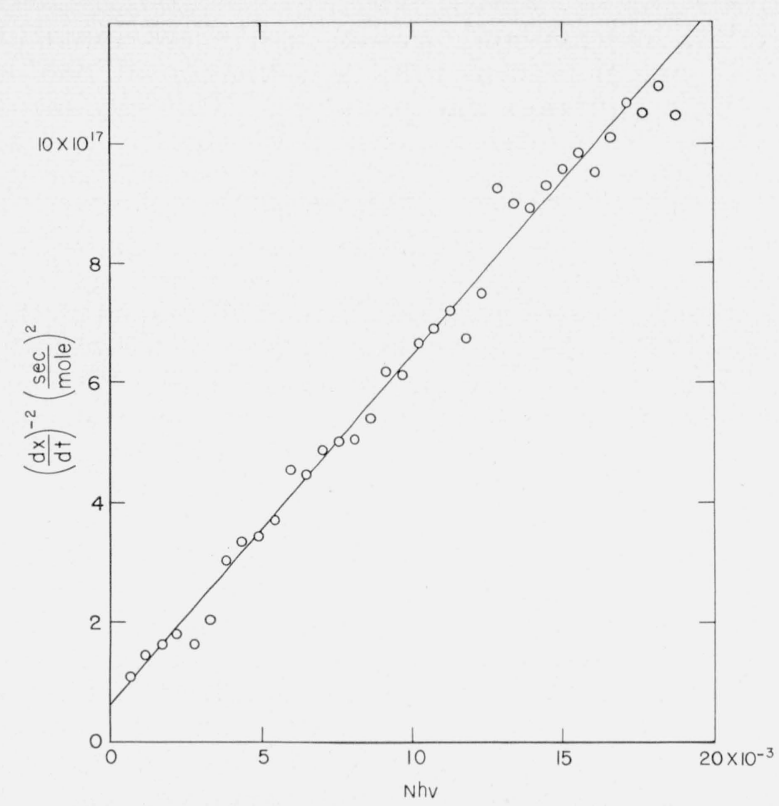

Figure 2. (Rate of $\mathrm{H}_{2}$ evolution) ${ }^{-2}$ versus dosage.

the calculation of quantum yields are probably accurate to within \pm 2 percent. Therefore $k=I_{a} \Phi_{\mathrm{H}}$ where $\Phi_{\mathrm{H}}$ is the initial quantum yield of hydrogen and $I_{a}$ is the intensity of absorbed light. It was assumed in the calculation of quantum yields that 43 percent of the light was absorbed. For a series of cellulose sheets of D. P. 1,710 containing initially $3.7 \times 10^{-6}$ equivalents of aldehyde per gram and $6.3 \times 10^{-6}$ equivalents of carboxyl per gram, $\Phi_{\mathrm{H}}=$ $0.0130 \mathrm{~mole} / \mathrm{Nh} \nu$ for the above intensities. A second series of sheets of D. P. 1,910 , containing $6.4 \times 10^{-6}$ aldehyde and $8.3 \times 10^{-6}$ carboxyl gave $\Phi_{\mathrm{H}}=0.0097$. The intersheet variation in $\Phi_{\mathrm{H}}$ was less than \pm 10 percent. Values of the constant, $a$, for the two series varied erratically between $6.0 \times 10^{6}$ and $10.0 \times 10^{6}$ moles $^{-1}$, and could not be correlated with intensity.

The rate of evolution of carbon monoxide plus carbon dioxide remained relatively constant. However, as can be seen from values obtained from a run at $9.62 \times 10^{-9} \mathrm{Nh \nu} / \mathrm{cm}^{2} \mathrm{sec}$ at $40^{\circ} \mathrm{C}$ in figure 3 , the rate of evolution of these gases decreases at first, passes through a minimum, and then steadily increases. The rate was proportional to the first power of the intensity, and the zero-order rate equation showed a combined quantum yield of carbon monoxide and carbon dioxide of $10.9 \times 10^{-4}$ moles/ Nhv for the first series of cellulose sheets and $8.0 \times 10^{-4}$ for the second series.

\subsection{Functional Groups}

Table 2 contains degree of polymerization (D. P.) and equivalents per gram of aldehyde and carboxyl groups for a series of cellulose sheets that received various dosages of $2537 \mathrm{~A}$ light at $40^{\circ} \mathrm{C}$.

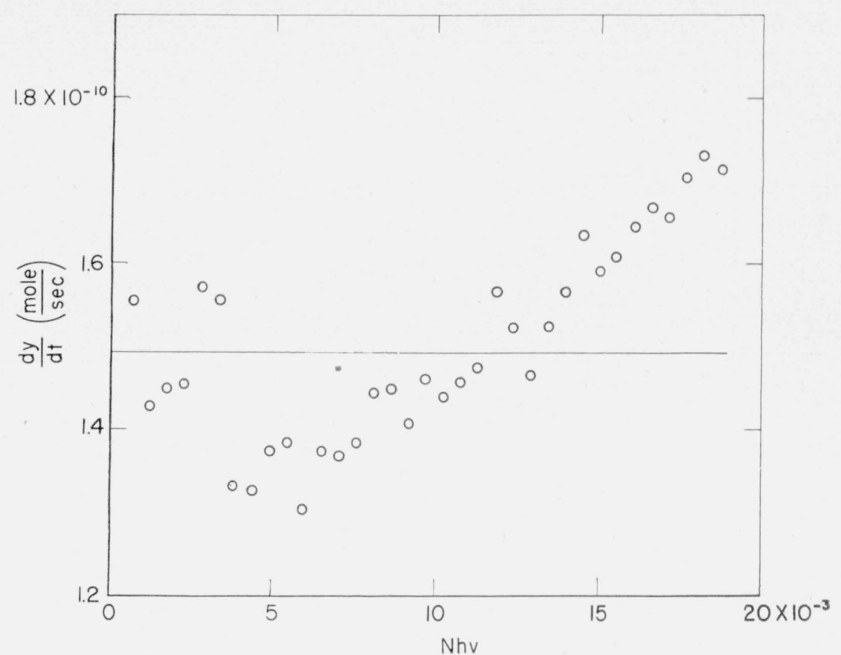

Figure 3. Rate of $\mathrm{CO}$ plus $\mathrm{CO}_{2}$ evolution versus dosage.

The D. P. in table 2 was calculated from an equation relating cuprammonium viscosity with weightaverage D. P. obtained from sedimentation data [13].

If the gradient in dosage follows Lambert's law, the number of chain scissions can be calculated from initial and final weight-average D. P.'s, the absorption coefficient, and the thickness of the sheet by assuming random fractures of an initially random distribution [14].

The absorption coefficient for unit thickness is 3.22 for the sheets described in table 2 .

Figure 4 contains the moles formed of aldehyde, carboxyl, hydrogen, carbon monoxide, and carbon dioxide, and chain fractures versus dosage of $2537 \mathrm{~A}$ light at $40^{\circ} \mathrm{C}$ for the 0.86 -g cellulose sheets described in table 2 .

Interruption of the irradiation by an evacuation for several days at $100^{\circ} \mathrm{C}$ had no effect on the kinetics of gaseous evolution.' A period of treatment with hydrogen followed by evacuation also had no effect. Treatment of the irradiated sample with oxygen followed by evacuation at $100^{\circ} \mathrm{C}$, however, caused a temporary inhibition of evolution of gas upon resumption of the irradiation, as shown in figure 5.

TABLE 2. Results of analyses of irradiated cellulose

\begin{tabular}{|c|c|c|c|}
\hline Dose & D. P. & RCHO & $\mathrm{RCOOH}$ \\
\hline $\begin{array}{l}\text { Nhv/g } \\
0.00 \times 10^{-3} \\
1.09 \\
2.18 \\
4.35\end{array}$ & $\begin{array}{l}1,710 \\
1,270 \\
1,200 \\
1,070\end{array}$ & $\begin{array}{l}\text { Moles } / g \\
0.37 \times 10^{-5} \\
1.43 \\
2.27 \\
2.70\end{array}$ & $\begin{array}{l}\text { Moles } / g \\
0.63 \times 10^{-5} \\
1.23 \\
1.51 \\
1.60\end{array}$ \\
\hline $\begin{array}{r}6.52 \\
9.57 \\
13.05 \\
19.58\end{array}$ & $\begin{array}{l}940 \\
870 \\
790 \\
690\end{array}$ & $\begin{array}{l}3.27 \\
4.46 \\
6.51 \\
\text { 8. } 47\end{array}$ & $\begin{array}{l}\text { 1. } 58 \\
2.11 \\
2.64 \\
2.65\end{array}$ \\
\hline
\end{tabular}




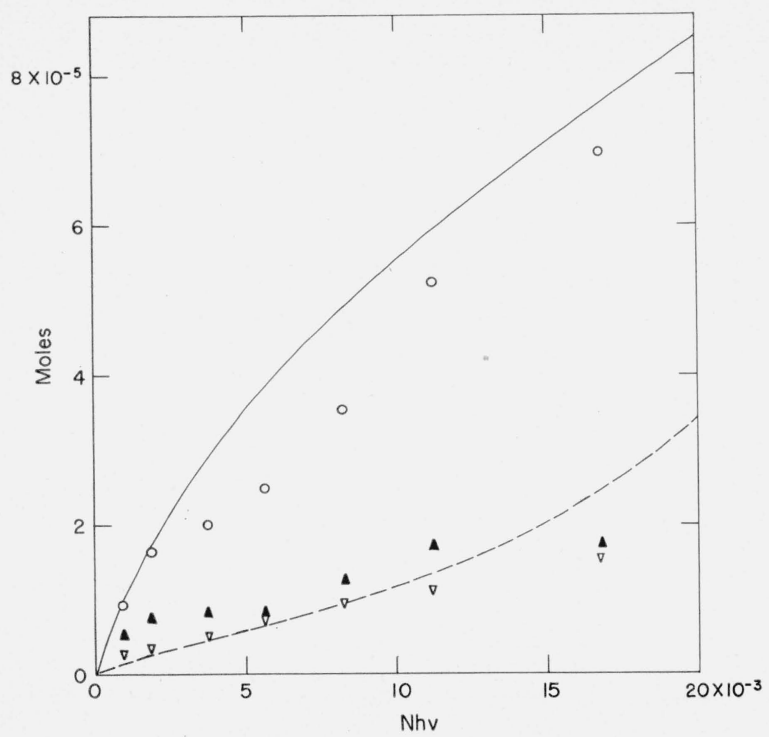

Figure 4. Moles of product versus dosage.

$\mathrm{O}$, Aldehyde; $\mathbf{\Lambda}$, carboxyl; $\nabla$, chain fracture; - - hydrogen; ...., $\mathrm{CO}$ and $\mathrm{CO}_{2}$.

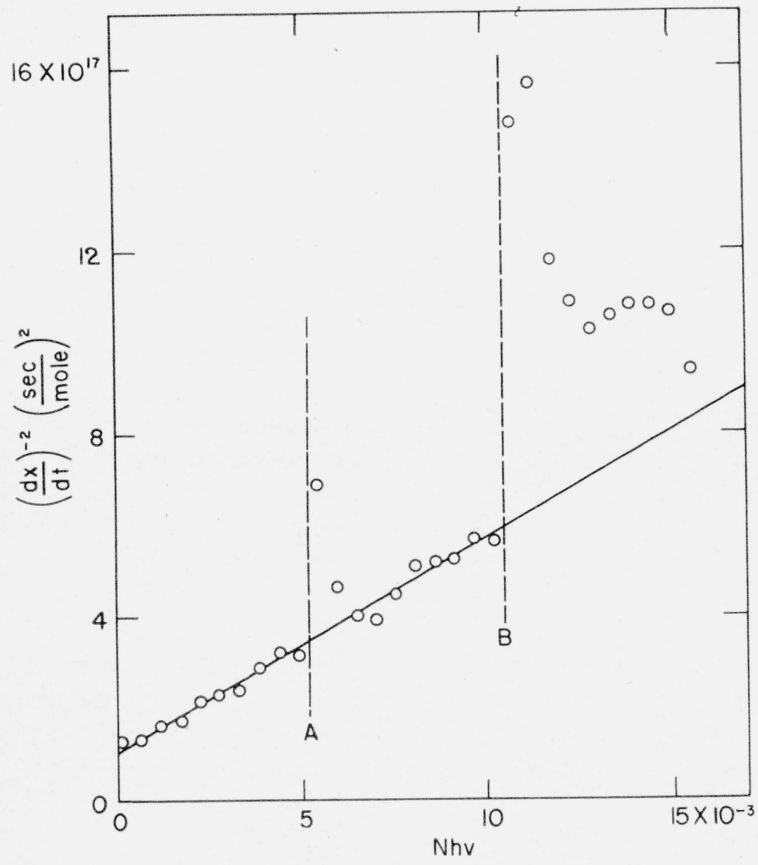

Figure 5. Effect of treatment with $\mathrm{H}_{2}$ and $\mathrm{O}_{2}$ on (rate of $\mathrm{H}_{2}$ evolution $)^{-2}$ versus dosage.

A, At this point the run was interrupted, $\mathrm{H}_{2}$ added, the sample heated at $100^{\circ}, \mathrm{C}$ for $6 \mathrm{hr}$, and evacuated at $100^{\circ} \mathrm{C}$ for 1 week before resumption of the run; B, as in $\mathrm{A}$, except $\mathrm{O}_{2}$ added instead of $\mathrm{H}_{2}$.

\section{Discussion}

The only direct measurement of the primary process in these experiments is the measurement of the rate of evolution of the gaseous products.

Before the measurement of change in carboxyl, aldehyde, and D. P., the irradiated specimens which contained oxygen-labile groups had been stored in air for several months until presumably all photosensitized oxidation was complete. The specimens may have been further modified by the fracture of alkali-sensitive linkages during the viscosity determinations and by the acidic reagents used in the carboxyl and aldehyde determinations. Since the more crystalline regions of the cellulose are not completely accessible to the latter two reagents, the carboxyl and aldehyde values may be low. However, a dosage of $0.02 N h \nu$ produced $8.5 \times 10^{-5}$ mole of hydrogen from a sample that contained initially $0.32 \times 10^{-5}$ equivalents of aldehyde and $0.54 \times 10^{-5}$ equivalents of carboxyl groups. Thus, even if the values for aldehyde and carboxyl are somewhat low, the amounts of aldehyde and carboxyl initially present are quite insufficient to account for the amount of hydrogen formed during the irradiation.

The evolution of hydrogen upon irradiation of cellulose with 2537 A light has not been noted by other investigators [2]. The prolonged degassing of the cellulose and the maintenance of a high vacuum during the irradiation apparently removed traces of water, adsorbed oxygen, or some other substance that either inhibited hydrogen formation directly or reacted with any hydrogen atoms produced before they could combine to form molecular hydrogen.

If hydrogen were formed by reactions involving the glucoside linkages, it would be necessary to postulate that these reactions do not involve chain scission because the ratio of chain scissions to molecules of hydrogen formed is 0.18 .

A plausible explanation of the source of hydrogen is the photolysis of alcohol groups by the over-all reaction in eq $(2)$

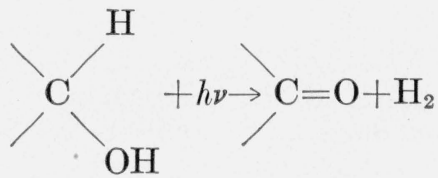

known to take place when simple alcohols are irradiated in the gas and liquid phases at below $2000 \mathrm{~A}$ $[15,16]$. Simple alcohols are transparent to $2537 \mathrm{~A}$ radiation.

Simple aldehydes and ketones fluoresce in the ultraviolet and are decomposed photochemically in the gas phase to produce carbon monoxide, various hydrocarbons, a little hydrogen, and, in the case of ketones, RCOCOR [17].

Although the reducing groups in cellulose may not be present as free aldehyde, these groups and any aldehyde groups formed from the irradiation of primary alcohol groups in the cellulose might be expected to react analogously to gas-phase photodecompositions as in eq (3). Likewise, keto groups from the photolysis of the secondary alcohol groups might react as in eq (4).

$$
\begin{gathered}
\mathrm{XCHO}+h \nu \rightarrow \mathrm{X} \cdot+\cdot \mathrm{CHO} \rightarrow \mathrm{CO}+\frac{1}{2} \mathrm{H}_{2}+\mathrm{X} . \\
\mathrm{XCO}+h \nu \rightarrow \dot{\mathrm{X}}-\dot{\mathrm{C}} \mathrm{O} \rightarrow \mathrm{CO}+\mathrm{X}:
\end{gathered}
$$


where $X$ represents the remaining cellulose chain which may contain free radicals or other labile groups.

The increasing carbonyl concentration from reaction (2) at the surface of the cellulose sheet would act as a nonvolatile filtering layer that would decrease the rate of photolysis of the alcohol groups. This effect can explain the product-inhibited rate equation for hydrogen evolution and the decreasing apparent quantum yields of hydrogen and functional groups as well as the increasing yield of carbon monoxide. Cellulose is known to fluoresce in the ultraviolet and the fluorescence increases upon irradiation [18].

Oxygen-labile linkages are formed during the irradiation of cellulose $[2,3]$; therefore, the close stoichiometric parallel to be seen in figure 4 between the calculated number of chain scissions and the amount of carboxyl formed indicates that the reactions involving chain fracture and oxidation to carboxyl in air may be closely related.

Washington, April 8, 1957.

\section{References}

[1] L. F. MeBurney, Cellulose and Cellulose Derivatives, Part I, Eds. E. Ott and H. M. Spurlin, p. 168 (Interscience Publishers, New York, N. Y., 1954).

[2] H. F. Launer and W. K. Wilson, J. Am. Chem. Soc. 71, 958 (1949).

[3] R. A. Stillings and R. J. Van Nostrand, J. Am. Chem. Soc. 66, 753 (1944).

[4] R. K. Warner and R. T. Mease, J. Research NBS 21, 609 (1938) RP1146.

[5] L. J. Heidt and H. B. Boyles, J. Am. Chem. Soc. 73, 5728 (1951)

[6] O. A. Battista, Ind. Eng. Chem., Anal. Ed. 16, 351 (1944).

[7] H. F. Launer and W. K. Wilson, Anal. Chem. 22, 455 (1950).

[8] G. F. Davidson, J. Text. Inst. 39, T65 (1948).

[9] W. K. Wilson and A. A. Padgett, Tappi 38, 292 (1955).

[10] H. F. Launer, W. K. Wilson, and J. H. Flynn, J. Research NBS 51, 237 (1953) RP2456.

[11] F. Benford, S. Schwartz, and G. Lloyd, J. Opt. Soc. Am. 38, 964 (1948).

[12] J. H. Flynn, J. Phys. Chem. 60, 1332 (1956).

[13] E. O. Kraemer, Ind. Eng. Chem. 30, 1200 (1938).

[14] J. H. Flynn, J. Polymer Sci. 27, 83 (1958).

[15] E. Patat, Z. Elektrochem 40, 425 (1934).

[16] L. Farkas and Y. Hirshberg, J. Am. Chem. Soc. 59, 2450 (1937).

[17] W. A. Noyes and P. A. Leighton, The Photochemistry of Gases, p. 336 (Reinhold Publishing Co., New York, N. Y., 1941).

[18] S. J. Lewis, J. Soc. Dyers Colourists 15, 29 (1924). 